\title{
Mathematical Modelling of Translation and Rotation Movement in Quad Tiltrotor
}

\author{
Andi Dharmawan ${ }^{\# *}$, Ahmad Ashari ${ }^{\#}$, Agfianto Eko Putra ${ }^{\# *}$ \\ ${ }^{\#}$ Department of Computer Science and Electronics, Faculty of Mathematics and Natural Science, \\ Universitas Gadjah Mada, Yogyakarta, Indonesia \\ E-mail:*andi_dharmawan@ugm.ac.id
}

\begin{abstract}
Quadrotor as one type of UAV (Unmanned Aerial Vehicle) is an underactuated mechanical system. It means that the system has some control inputs is lower than its DOF (Degrees of Freedom). This condition causes quadrotor to have limited mobility because of its inherent under actuation, namely, the availability of four independent control signals (four-speed rotating propellers) versus 6 degrees of freedom parameterizing quadrotor position or orientation in space. If a quadrotor is made to have 6 DOF, a full motion control system to optimize the flight will be different from before. So it becomes necessary to develop over actuated quad tiltrotor. Quad tiltrotor has control signals more than its DOF. Therefore, we can refer it to the overactuated system. We need a good control system to fly the quad tiltrotor. Good control systems can be designed using the model of the quad tiltrotor system. We can create quad tiltrotor model using its dynamics based on Newton-Euler approach. After we have a set of model, we can simulate the control system using some control method. There are several control methods that we can use in the quad tiltrotor flight system. However, we can improve the control by implementing a modern control system that uses the concept of state space. The simulations show that the quad tiltrotor has done successful translational motion without significant interference. Also, undesirable rotation movement in the quad tiltrotor flight when performing the translational motions resulting from the transition process associated with the tilt rotor change was successfully reduced below 1 degree.
\end{abstract}

Keywords: UAV; overactuated; control; state space

\section{INTRODUCTION}

UAV (Unmanned Aerial Vehicle) or UAS (Unmanned Aircraft System) is defined as a plane without a pilot sitting in it, uses aerodynamic forces to fly, either standalone (autonomous) with the help of auto pilot or piloted remotely and can carry a payload (payload) or not [1]. UAVs have been developed for a variety of purposes and missions from having the ability to perform various types of sensing missions either for civilian or military to monitor the building. The missions consist of surveillance, reconnaissance, monitoring, air patrols, high-resolution aerial photography and so forth [2].

UAV is a mechanical system that underactuated. It means a system has a lower number of actuators than degrees of freedom (DOF). In this case, the system is said to be trivially underactuated [3]. However, the design of that underactuated Quadrotor still limits the ability to fly in space that is free or irregular and also lowers the possibility of interacting with the environment by deploying the desired style-free direction.
Motivated by these considerations, several solutions have been proposed in previous studies that cover different concepts, for example, the mechanism of tilt-wing [4], UAV that uses a non-parallel direction of the lift force (but fixed) [5], or implementing the tiltrotor system [6]. The possibility of combining several modules of underactuated rotorcraft to achieve full movement of the 6-DOF on a UAV is theoretically still being explored. This issue focuses on the optimal allocation of available control inputs (that may be excessive) [7]. On the contrary, the possibility of the swinging lifting force of quadrotor where the main direction of the lift force (2 DOF), which can be actively regulated may be considered [8].

Also, if the UAV made it to the movement of the full 6 DOF or more, control systems that are used to optimise the movement of UAV flight becomes different to those previously applied. One thing that needs to be optimised is the attitude control of the UAV flight. 


\section{MATERIAL AND METHOD}

Multirotor is a popular topic developed by many researchers [9], [10], and [11]. Multirotor can fly vertical take-off and landing (VTOL) compared to fixed wing aircraft types [12]. Because of these advantages, it can be used almost anywhere on a land.

Quad tiltrotor as the development of the multirotor has several advantages that are not owned by ordinary multirotor. One is the ability to move horizontal translations without forming pitch and roll angles. This translational movement is performed by rotating the rotor toward the desired translational motion. During the translational movement progresses, the system needs an anti-rotation motion to avoid unwanted rotation movement. Therefore, a translational and rotational motion model of the quad tiltrotor is required to determine the appropriate control system of the system.

\section{A. Quad Tiltrotor Kinematics}

We can model a quad tiltrotor as a connection of five main rigid bodies in relative motion between the quad tiltrotor body $\boldsymbol{B}$ and 4 groups' propeller $\boldsymbol{P}_{i}$. It consists of 4 arm motor for swinging the actuation mechanism of each propeller. The propellers itself are connected to each rotor of the motor for rotating propellers actuation (shown in Fig. 1).

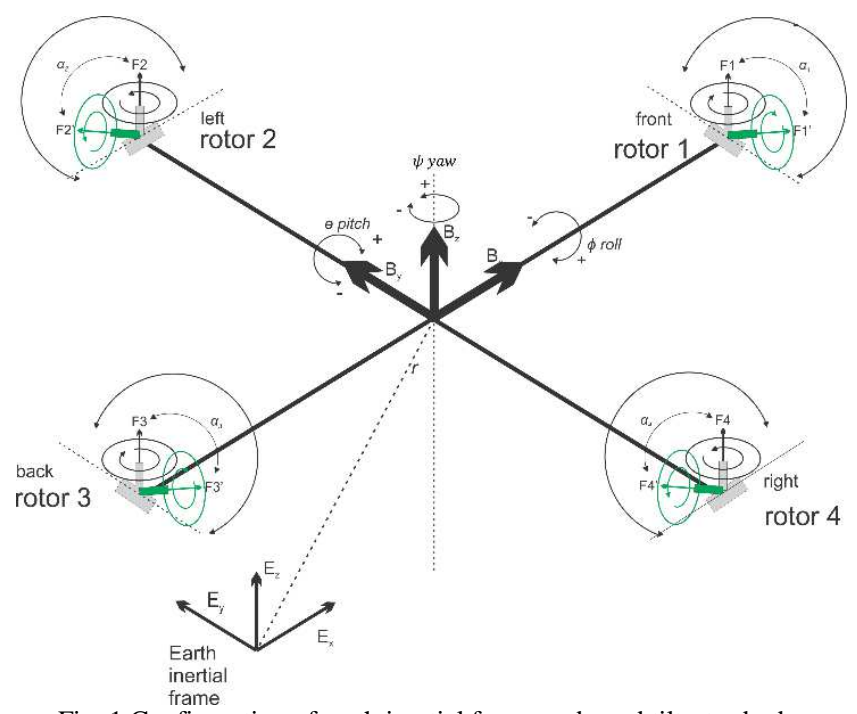

Fig. 1 Configuration of earth inertial frame and quad tiltrotor body.

Suppose $\boldsymbol{E}:\left\{\boldsymbol{O}_{E} ; \boldsymbol{E}_{\mathbf{X}} \boldsymbol{E}_{y,} \boldsymbol{E}_{z}\right\}$ is an inertial frame of the earth, $\boldsymbol{B}:\left\{\boldsymbol{O}_{B} ; \boldsymbol{B}_{x}, \boldsymbol{B}_{y}, \boldsymbol{B}_{z}\right\}$ is a moving frame that mounted on the body of quad tiltrotor at the center of mass, and $\boldsymbol{P}_{i}:\left\{\boldsymbol{O}_{P_{i}} ; \boldsymbol{P}_{i_{x}}, \boldsymbol{P}_{i_{y}}, \boldsymbol{P}_{i_{z}}\right\}(i=1, \ldots, 4)$ is defined as the frame that is connected to the propeller group $i$-th as shown in Fig. 2.

To determine the position of the quad tiltrotor, we can use the rotation matrix $\boldsymbol{R} . \boldsymbol{R} \in S O(3)$ is a rotation matrix representing the orientation of the second frame (body) on the first frame (of the earth), that is shown in (1),

$\boldsymbol{R}(\phi, \theta, \psi)=\left[\begin{array}{ccc}c \psi c \theta & -s \psi c \phi+c \psi s \theta s \phi & s \psi s \phi-c \psi s \theta c \phi \\ s \psi c \theta & c \psi c \phi+s \psi s \theta s \phi & -c \psi s \phi+s \psi s \theta c \phi \\ -s \theta & c \theta s \phi & c \phi c \theta\end{array}\right]$

where $c=\cos$, and $s=\sin$.

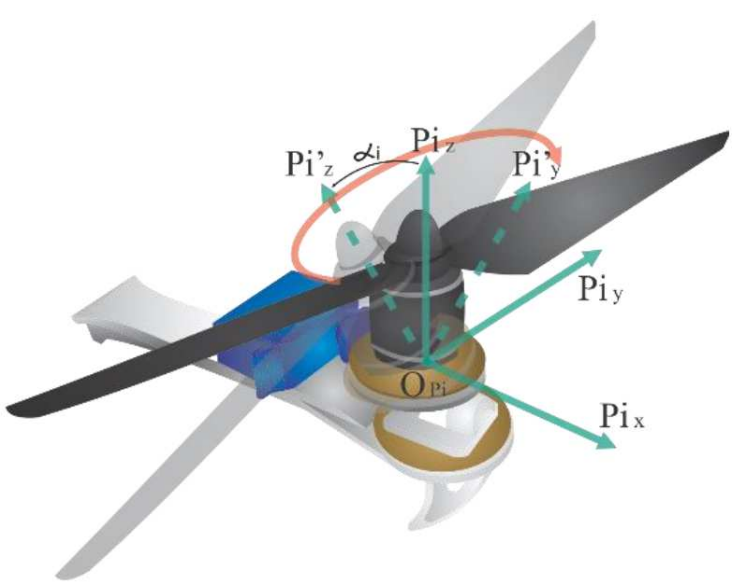

Fig. 2 The $\boldsymbol{i}$-th swinging arm visualizes the body frame $\boldsymbol{P}_{i}$, propellers associated with lifting force $F_{i}$, and the angle of swinging propellers $\boldsymbol{\alpha}_{i}$

Pitch $(\theta)$ rotation of quad tiltrotor will occur if we modify propeller 1 and 3 speeds. Changing the speed of the propeller 2 and 4 where the propeller 2 is faster than the propeller 4 or otherwise will produce roll $(\phi)$ rotation. Yaw $(\psi)$ rotation is softer than the other rotations because it is a result of the counter-torque difference between each pair of blades. We can control yaw $(\psi)$ rotation on the $B_{z}$ axis by varying the speed of the pair propeller on the opposite shaft.
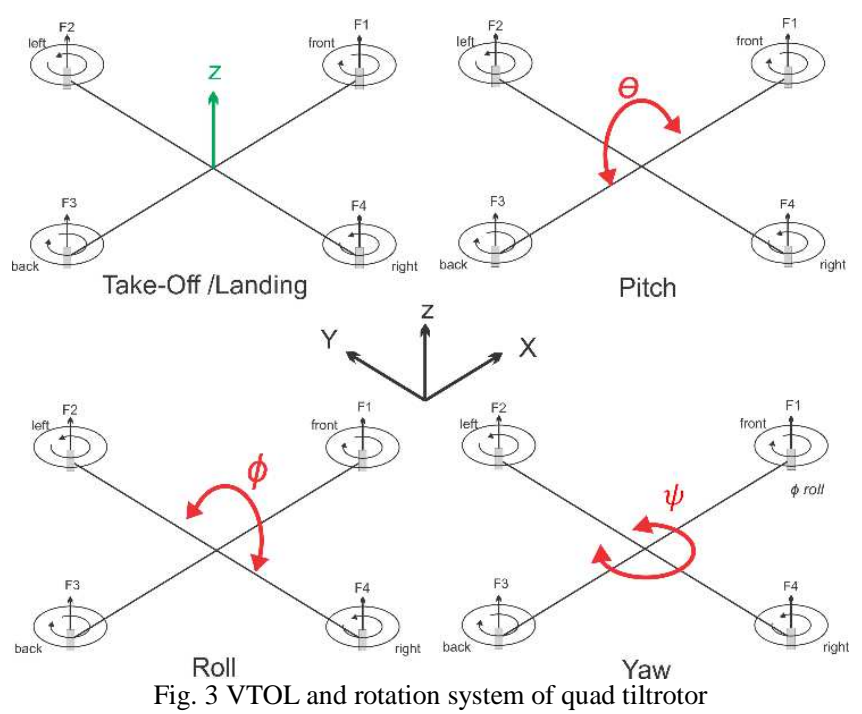

${ }^{E} \boldsymbol{R}_{B}$ will represent the orientation of the body frame associated with the frame of the earth, while ${ }^{B} \boldsymbol{R}_{P_{i}}$ shows the orientation of the $i$-th propellers frame group that is connected to the body frame. By showing $\alpha_{i} \in \mathbb{R}$ which is propellers swinging angle to the $\boldsymbol{P}_{i_{x}}$-axis, then in accordance with Fig. 2:

$$
{ }^{\bar{s}} R_{F_{i}}=R_{Z}\left((i-1) \frac{\pi}{2}\right) R_{x}\left(\kappa_{i}\right), \quad i=1, \ldots, 4
$$$$
\text { where } \boldsymbol{R}_{x}\left(\alpha_{i}\right)=\left[\begin{array}{ccc}
1 & 0 & 0 \\
0 & \cos \alpha_{i} & -\sin \alpha_{i} \\
0 & \sin \alpha_{i} & \cos \alpha_{i}
\end{array}\right]
$$$$
\boldsymbol{R}_{z}(\gamma)=\left[\begin{array}{ccc}
\cos \gamma & -\sin \gamma & 0 \\
\sin \gamma & \cos \gamma & 0 \\
0 & 0 & 1
\end{array}\right], \quad \alpha_{i} \text { is the } i \text {-th group }
$$
propeller tilt angle, and $\gamma=(i-1) \frac{\pi}{2}$. 
In the same way, let

$$
{ }^{B} \boldsymbol{O}_{P_{i}}=\boldsymbol{R}_{Z}\left((i-1) \frac{\pi}{2}\right)\left[\begin{array}{l}
l \\
0 \\
0
\end{array}\right], \quad i=1, \ldots, 4 \text {. }
$$

is the starting point of the propellers frame $\boldsymbol{P}_{i}$ on the frame body with $l$ is the distance $\boldsymbol{O}_{P_{i}}$ from $\boldsymbol{O}_{B}$ [13].

Therefore, the configuration quad tiltrotor is fully influenced by the position of the body $\boldsymbol{p}={ }^{E} \boldsymbol{O}_{B} \in \mathbb{R}^{3}$ and the orientation ${ }^{E} \boldsymbol{R}_{B}$ on the earth frame. The all four angles of swing $\alpha_{i}$ specify the orientation of the propellers group on the body frame (rotation against $\boldsymbol{P}_{i_{x}}$ ). Propeller rotation angle to $\boldsymbol{P}_{i_{z}}$ is eliminated as a configuration variable, although the propeller rotating speed $\bar{\omega}_{i}$ to $\boldsymbol{P}_{i_{z}}$ will be part of the system model.

\section{B. Quad Tiltrotor Dynamics}

In the process of determining the model of flying objects such a quad tilt-rotor, we can use Newton-Euler approach. Newton-Euler describes the combined translational and rotational dynamics of a rigid body. For a quad tiltrotor system, we can begin to determine the system model for the translation and rotation movements.

Newton-Euler consists of the concept of Newton's Second Law and Euler's Second Law. We can use Newton's second law which is a relation of force $\boldsymbol{F}$ and acceleration $\boldsymbol{a}$ experienced a centre of mass, as shown in equation (4). We can use it to determine the model of translational movement.

$$
\boldsymbol{F}=m \boldsymbol{a}
$$

The force experienced by quad tiltrotor is the resultant of the thrust generated by each motor and the gravity force, as shown in equation (5),

$$
\boldsymbol{F}={ }^{E} \boldsymbol{R}_{B_{g}} F_{T}+\boldsymbol{F}_{g}
$$

where ${ }^{E} \boldsymbol{R}_{B_{z}}$ is rotation matrix in body axis $B_{z}$ to earth axis $\boldsymbol{E}$ (third column in rotation matrix $\boldsymbol{R}$ as shown in (1)).

Total lift force $F_{T}$ as a resultant of each brushless motor thrust can be obtained using (6)

$$
F_{T}=\sum_{i=1}^{4} F_{i}=\sum_{i=1}^{4} b_{i} \omega_{i}^{2}, i=1,2,3,4
$$

where $F_{i}$ is the $i$-th brushless motor thrust, $b_{i}$ is ith brushless motor thrust constant, and $\omega_{i}$ is ith brushless motor angular velocity. We can determine brushless motor thrust $F$ by using the equation(7) [14].

$$
F=C_{F} \rho A R^{2} \omega^{2}
$$

where $C_{F}$ is non-dimensional thrust constant, $\rho$ is air density $\left(1,184 \mathrm{~kg} / \mathrm{m}^{3}\right), A$ is an area formed by the propeller when turning, $R$ is the radius of the propeller, and $\omega$ is the angular velocity of the propeller ( $\mathrm{deg} / \mathrm{sec}$ ). By using equation (6) and (7), then the motor thrust constant $b$ shown in equation (8).

$$
b=C_{F} \rho A R^{2}
$$

Gravitation force $\boldsymbol{F}_{g}$ is shown in (9).

$$
\boldsymbol{F}_{g}=\left[\begin{array}{c}
0 \\
0 \\
-m g
\end{array}\right]
$$

where $m$ is the total mass of quad tiltrotor measured at the center of gravity (CoG) and $g$ is earth gravity $\left(9.8 \mathrm{~m} / \mathrm{s}^{2}\right)$.

Quad tiltrotor has a translational motion on the vertical Earth's axis $E_{z}$ like shown in equation (10).

$$
\begin{gathered}
F_{z}=E_{z} R_{B_{g}} F_{T}-m g \\
m z=(\cos \phi \cos \theta) F_{T}-m g
\end{gathered}
$$

We can apply this equation if and only if the vertical body axis $B_{z}$ of quad tiltrotor parallel to the vertical Earth's axis $E_{z}$. In this condition, the tilt angle $\alpha_{i}$ on each propeller group is 0 .

However, if the tilt angle $\alpha_{i}>0$, then using equation (2), the total force $F_{T}$ in equation (6) becomes:

$$
F_{T_{z}}=\sum_{i=1}^{4} B_{z} R_{P_{i_{z}}} F_{i}
$$

Then we combine equation (11) with equation (10) which becomes:

$$
m \ddot{z}=(\cos \phi \cos \theta) F_{T_{z}}-m g
$$

where $m$ is the quad tiltrotor total mass.

Quad tiltrotor has several modes to perform a translational motion in the lateral axis $\left(E_{x}\right)$ and the longitudinal axis $\left(E_{y}\right)$. They consist of:

1. Quad tiltrotor moves horizontally based on the magnitude of the pitch angle $(\theta)$ and the roll angle $(\phi)$ formed by the plane towards the Earth's axis $E$ $(|\theta|>0,|\phi|>0)$, as shown in equation (13) and (14). Each of the group of propellers axis $P_{i_{z}}$ aligned with the axis of the vertical body axis $B_{z}\left(\alpha_{i}=0\right)$.

- Force in the $x$-axis:

$$
F_{x}=E_{x} R_{B_{z}} F_{T}+0
$$$$
m \ddot{x}=(\sin \psi \sin \phi-\cos \psi \sin \theta \cos \phi) F_{T}
$$

- Force in the $y$-axis:

$$
\begin{gathered}
F_{y}=E_{y} R_{B_{x}} F_{T}+0 \\
m \ddot{y}=(-\cos \psi \sin \phi+\sin \psi \sin \theta \cos \phi) F_{T}
\end{gathered}
$$

2. Translational motion occurs by using changes in tilt angle from each group propeller. However, the internal forces do not cause the plane makes an angle of pitch $(\theta)$ and roll angle $(\varphi)$ to the axis of the earth $(\theta=0, \phi=0)$. For this mode, we can determine the translational motion along $E_{x}$ axis and $E_{y}$ axis by modifying the equation (13) and (14) into the equation (15) and (16).

$$
\begin{gathered}
F_{T_{x}}=\sum_{i=1}^{4}{ }^{B_{z}} R_{P_{i x}} F_{i} \\
m \ddot{x}=F_{T_{x}} \\
F_{T_{y}}=\sum_{\substack{i=1 \\
m y=F_{T_{y}}}}^{B_{z} R_{P_{i y}} F_{i}}
\end{gathered}
$$

3. Translational motion occurs by using the change tilt angle $(|\alpha|>0)$ and the internal forces cause the aircraft to form pitch angle $(\theta)$ and the roll angle $(\phi)$ to the axis of the earth $(|\theta|>0,|\phi|>0)$. For this mode, we can determine the translational motion along the axis $E_{x}$ and $E_{y}$ by modifying the equation (1), (15), and (16), into the equation (17) and (18).

$$
\begin{gathered}
m \dot{x}=E_{x} R_{B_{x}} F_{T_{x}} \\
m \ddot{x}=(\cos \psi \cos \theta) F_{T_{x}} \\
m \ddot{y}=E_{y R_{B_{y}} F_{T_{y}}} \\
m \dot{y}=(\cos \psi \cos \phi+\sin \psi \sin \theta \sin \phi) F_{T_{y}}
\end{gathered}
$$


In the first mode, the quad tiltrotor rotation around the $x$ and $y$-axis can be determined by using equation (19) and (20).

- Torque around the $x$-axis (roll rotation):

$$
\begin{gathered}
\tau_{\phi}=I_{x x} \ddot{\phi} \\
\tau_{\phi}=\left(F_{2}-F_{4}\right) l \\
I_{x x} \ddot{\phi}=\left(F_{2}-F_{4}\right) l
\end{gathered}
$$

- Torque around the $y$-axis (pitch rotation):

$$
\begin{gathered}
\tau_{\theta}=I_{y y} \ddot{\theta} \\
\tau_{\theta}=\left(F_{1}-F_{3}\right) l \\
I_{y y} \ddot{\theta}=\left(F_{1}-F_{3}\right) l
\end{gathered}
$$

where $l$ is the distance between the rotation axis to the thrust, $\phi$ is the roll angle, and $\theta$ is the pitch angle.

Each of the group of propellers axis $P_{i_{z}}$ aligns with the axis of the body $B_{z}$, so tilting angle $\alpha_{i}=0$. In the second and third mode, the rotational motion of quad tiltrotor around the $x$ and $y$-axes can also be caused by rotors rotation torque effects that experiencing tilting. Therefore, by using equation (19), (20), (15), and (16), we can determine the rotational motion around the $x$ and $y$-axes as shown in equation (21) and (22).

$$
\begin{aligned}
& \tau_{\phi}=I_{x x} \ddot{\phi} \\
& \tau_{\phi}=\left({ }^{B_{x}} R_{P_{x_{z}}} F_{2}-{ }^{B_{z}} R_{P_{4_{z}}} F_{4}\right) l+{ }^{B_{x}} R_{P_{2 x}} \tau_{2}+{ }^{B_{x}} R_{P_{4_{z}}} \tau_{4} \\
& I_{x x} \ddot{\phi}=\left(B_{z} R_{P_{2 x}} F_{2}-B_{z} R_{P_{4_{z}}} F_{4}\right) l+B_{z} R_{P_{2 x}} \tau_{2}+B_{x} R_{P_{4_{z}}} \tau_{4} \\
& \tau_{\theta}=I_{y y} \ddot{\theta} \\
& \tau_{\theta}=\left({ }^{B_{z}} R_{P_{1_{z}}} F_{1}-{ }^{B_{z}} R_{P_{z_{z}}} F_{3}\right) l+{ }^{B_{y}} R_{P_{1_{z}}} \tau_{1}+{ }^{B}{ }_{R_{P_{3_{z}}}} \tau_{3} \\
& I_{y y} \ddot{\theta}=\left(B_{x} R_{P_{1_{z}}} F_{1}-B_{x} R_{P_{3_{z}}} F_{3}\right) l+{ }^{B} R_{P_{1_{z}}} \tau_{1}+{ }^{y_{y}} R_{P_{3_{z}}} \tau_{3}
\end{aligned}
$$

The system automatically sets tilting the angle $\alpha_{i}$ based on the equation (23).

$$
\begin{gathered}
{\left[\begin{array}{l}
\alpha_{x} \\
\alpha_{y}
\end{array}\right]=\left[\begin{array}{r}
\operatorname{atan}\left(\frac{\frac{F_{x}}{2}}{\frac{F_{z}}{4}}\right) \\
\left(\frac{F_{y}}{\frac{2}{F_{z}}}\right) \\
\frac{\operatorname{atan}}{4}
\end{array}\right]=\left[\begin{array}{r}
\operatorname{atan}\left(\frac{4 F_{z}}{2 F_{z}}\right) \\
\operatorname{atan}\left(\frac{4 F_{y}}{2 F_{z}}\right)
\end{array}\right]=\left[\begin{array}{l}
\operatorname{atan}\left(2 \frac{F_{x}}{F_{z}}\right) \\
\operatorname{atan}\left(2 \frac{F_{y}}{F_{z}}\right)
\end{array}\right]} \\
{\left[\begin{array}{l}
\alpha_{1} \\
\alpha_{2} \\
\alpha_{3} \\
\alpha_{4}
\end{array}\right]=\left[\begin{array}{ll}
0 & 1 \\
1 & 0 \\
0 & 1 \\
1 & 0
\end{array}\right]\left[\begin{array}{l}
\alpha_{x} \\
\alpha_{y}
\end{array}\right]}
\end{gathered}
$$

For rotation motion, we can use Euler's Second Law. That explains the relationship of torque $\boldsymbol{\tau}$ and the angular acceleration $\boldsymbol{\alpha}$ as shown in equation (24),

$$
\tau=I_{c m} \alpha
$$

where

$$
\boldsymbol{I}_{c m}=\left[\begin{array}{ccc}
I_{x x} & 0 & 0 \\
0 & I_{y y} & 0 \\
0 & 0 & I_{z z}
\end{array}\right]
$$

$I_{x x}$ is a moment of inertia in the $x$-axis of the body, $I_{y y}$ is a moment of inertia in the $y$-axis of the body, and $I_{z z}$ is a moment of inertia in the $z$-axis of the body. We can determine the values of each $I_{x x^{\prime}} I_{y y^{\prime}}$ dan $I_{z z}$ by using (26), (27), and (28).

$$
I_{x x}=\sum_{j=1}^{n}\left(I_{G_{x x_{j}}}+m_{j}\left(y_{j}^{2}+z_{j}^{2}\right)\right)
$$

$$
\begin{aligned}
& I_{y y}=\sum_{j=1}^{n}\left(I_{G_{y y_{j}}}+m_{j}\left(x_{j}^{2}+z_{j}^{2}\right)\right) \\
& I_{z z}=\sum_{j=1}^{n}\left(I_{G_{z a_{j}}}+m_{j}\left(x_{j}^{2}+y_{j}^{2}\right)\right)
\end{aligned}
$$

where $I_{G_{x x_{j}}}, I_{G_{y y_{j}}}$ and $I_{G_{z z_{j}}}$ are the moment of inertia in $x, y$, and $z$-axis of the $j$ th quad tiltrotor component body respectively, and $z_{j}$ denote the position vector of the $j$ th quad tiltrotor component $\mathrm{CoG}$ about a fixed inertial frame, and $m_{j}$ is the $j$ th quad tiltrotor component mass [15].

By using thrust force $\boldsymbol{F}$ and the distance between the axes of rotation with the thrust force, then we can determine the torque $\tau$ by following (29)

$$
\boldsymbol{\tau}=\boldsymbol{F} r
$$

where $r$ is the distance between the axes of rotation with the thrust force $\boldsymbol{F}$.

For rotational motion around the vertical axis $B_{z}$, quad tiltrotor has the mathematical model as shown in equation (30), if and only if the tilt angle of each group $\alpha_{i}$ propeller is 0 .

- Torque around the $z$-axis (yaw rotation):

$$
\begin{gathered}
\tau_{\psi}=I_{z z} \ddot{\psi} \\
\tau_{\psi}=\tau_{1}-\tau_{2}+\tau_{3}-\tau_{4} \\
I_{z z} \ddot{\psi}=\tau_{1}-\tau_{2}+\tau_{3}-\tau_{4}
\end{gathered}
$$

where $\tau_{i}$ is the torque of the $i$ th brushless motor and $\psi$ is the yaw angle.

We can determine brushless motor torque $\tau$ using (31) [14].

$$
\tau=C_{\tau} \rho A R^{3} \omega^{2}
$$

where $C_{\tau}$ is a non-dimensional constant torque, $\rho$ is the density of air $\left(1.184 \mathrm{~kg} / \mathrm{m}^{3}\right), A$ is the area which is formed from the propeller when spinning, $R$ is the radius quadrotor measured from the midpoint of the center of mass to the midpoint of the brushless motor, and $\omega$ is the rotational speed of the propeller ( $\mathrm{rad} / \mathrm{s})$. Hence, the brushless motor torque constant $k$ is shown in equation (32).

$$
k=C_{T} \rho A R^{2}
$$

If $\alpha_{i}>0$, then the thrust generated by the rotor tilting can influence the yaw rotational motion of the quad tiltrotor. Therefore we convert equation (30) into the equation (33).

$$
\begin{aligned}
& \tau_{\psi}=I_{z z} \ddot{\psi} \\
& \tau_{\psi}={ }^{B} R_{P_{1_{z}}} \tau_{1}-{ }^{B_{z}} R_{P_{2_{z}}} \tau_{2}+{ }^{B_{z}} R_{P_{3_{z}}} \tau_{3}-{ }^{B_{z}} R_{P_{4_{z}}} \tau_{4}+{ }^{B_{x}} R_{P_{4_{z}}} F_{4} \\
& -{ }^{B} R_{P_{2_{z}}} F_{2}+{ }^{B_{y}} R_{P_{3 z}} F_{3}-{ }^{B_{y}} R_{P_{1 z}} F_{1} \\
& I_{z z} \ddot{\psi}={ }^{B_{z}} R_{P_{1}} \tau_{1}-{ }^{B_{z}} R_{P_{2_{z}}} \tau_{2}+{ }^{B_{z}} R_{P_{3_{z}}} \tau_{3}-{ }^{B_{z}} R_{P_{4 z}} \tau_{4}+{ }^{B_{x}} R_{P_{4_{z}}} F_{4} \\
& -{ }^{B_{x}} R_{P_{2_{Z}}} F_{2}+{ }^{B_{y}} R_{P_{3_{Z}}} F_{3}-{ }^{B_{y}} R_{P_{1_{z}}} F_{1} \\
& \text { battery }
\end{aligned}
$$

Fig. 4 Quad tiltrotor side view design

Equation (34) and (35) show the moment of inertia in each of the $x$ and $y$-axes on the quad tiltrotor by using equation (26) and (27). $I_{z z}$ is the moment of inertia on the $\mathrm{z}$ axis can be determined using equation (28) so that it becomes equation (36). 


$$
\begin{aligned}
& I_{x x}=\left(\sum_{i=1}^{4}\left(\frac{1}{12} M_{B M}\left(3 r_{B M}^{2}+h_{B M}^{2}\right)+M_{B M}\left(y_{B M_{i}}^{2}+z_{B M_{i}}^{2}\right)\right)\right) \\
& +\left(\sum _ { i = 1 } ^ { 4 } \left(\frac{1}{12} M_{B M M}\left(3 r_{B M M}^{2}+h_{B M M}^{2}\right)\right.\right. \\
& \left.\left.+M_{B M M_{i}}\left(y_{B M M_{i}}^{2}+z_{B M M_{i}}^{2}\right)\right)\right) \\
& +\left(\sum _ { i = 1 } ^ { 4 } \left(\frac{1}{12} M_{P}\left(3 r_{P}^{2}+h_{P}^{2}\right)\right.\right. \\
& \left.+M_{P}\left(y_{P_{i}}^{2}+z_{P_{i}}^{2}\right)^{2}\right) \\
& +2\left(\frac{1}{12} M_{E}\left(h_{E}^{2}+l_{E}^{2}\right)\right. \\
& \left.+M_{E}\left(\frac{1}{2} h_{L}+\frac{1}{2} h_{E}\right)^{2}\right) \\
& +2\left(\frac{1}{12} M_{E}\left(h_{E}^{2}+w_{E}^{2}\right)\right. \\
& +M_{E}\left(\left(\frac{1}{2} h_{L}+\frac{1}{2} h_{E}\right)^{2}\right. \\
& \left.\left.+\left(\frac{1}{2} l_{L}+\frac{1}{2} s_{C B}\right)^{2}\right)\right) \\
& +2\left(\frac{1}{12} M_{S M}\left(h_{S M}^{2}+l_{S M}^{2}\right)\right. \\
& \left.+M_{S M}\left(\frac{1}{2} h_{L}+\frac{1}{2} h_{S M}\right)^{2}\right) \\
& +2\left(\frac{1}{12} M_{S M}\left(h_{S M}^{2}+w_{S M}^{2}\right)\right. \\
& +M_{S M}\left(\left(\frac{1}{2} h_{L}+\frac{1}{2} h_{S M}\right)^{2}\right. \\
& \left.\left.+\left(\frac{1}{2} s_{C B}+l_{L}-\frac{1}{2} w_{S M}\right)^{2}\right)\right) \\
& +2\left(\frac{1}{12} M_{L}\left(h_{L}^{2}+l_{L}^{2}\right)\right) \\
& +2\left(\frac{1}{12} M_{L}\left(h_{L}^{2}+w_{L}^{2}\right)\right. \\
& \left.+M_{L}\left(\left(\frac{1}{2} l_{L}+\frac{1}{2} s_{C B}\right)^{2}\right)\right) \\
& +\left(\frac{1}{12} M_{C B}\left(h_{C B}^{2}+s_{C B}^{2}\right)\right) \\
& +\left(\frac{1}{12} M_{B}\left(h_{B}^{2}+l_{B}^{2}\right)\right. \\
& \left.+M_{B}\left(\frac{1}{2} h_{C B}+\frac{1}{2} h_{B}\right)^{2}\right)
\end{aligned}
$$

where $M_{B M}$ is brushless motor mass, $r_{B M}$ is brushless motor radius, $h_{B M}$ is brushless motor height, $M_{B M M}$ is the brushless motor mounting mass, $r_{B M M}$ is the brushless motor mounting radius, $h_{B M M}$ is brushless motor mounting height, $M_{S M}$ is servo motor mass, $r_{S M}$ is servo motor radius, $h_{S M}$ is servo motor height, $M_{P}$ is propeller mass, $r_{P}$ is propeller radius, $h_{p}$ is propeller height, $M_{E}$ is ESC mass, $l_{E}$ is ESC length, $w_{E}$ is ESC width, $h_{E}$ is ESC height, $M_{L}$ is quad tiltrotor arm mass, $l_{L}$ is quad tiltrotor arm length, $w_{L}$ is quad tiltrotor arm width, $h_{L}$ is quad tiltrotor arm height, $M_{C B}$ is center box mass, $s_{C B}$ is center box side length, $h_{C B}$ is center box height, $M_{B}$ is battery mass, $l_{B}$ is battery length, $w_{B}$ is battery width, and $h_{B}$ is battery height. Details of each component are shown in Fig. 4 and Fig. 5.

$$
\begin{aligned}
& I_{y y}=\left(\sum_{i=1}^{4}\left(\frac{1}{12} M_{B M}\left(3 r_{B M}^{2}+h_{B M}^{2}\right)+M_{B M_{i}}\left(x_{B M_{i}}^{2}+z_{B M_{i}}^{2}\right)\right)\right) \\
& +\left(\sum _ { i = 1 } ^ { 4 } \left(\frac{1}{12} M_{B M M}\left(3 r_{B M M}^{2}+h_{B M M}^{2}\right)\right.\right. \\
& \left.\left.+M_{B M M_{i}}\left(x_{B M M_{i}}^{2}+z_{B M M_{i}}^{2}\right)\right)\right) \\
& +\left(\sum _ { i = 1 } ^ { 4 } \left(\frac{1}{12} M_{P}\left(3 r_{P}^{2}+h_{P}^{2}\right)\right.\right. \\
& \left.+M_{P}\left(x_{P_{i}}^{2}+z_{P_{i}}^{2}\right)^{2}\right) \\
& +2\left(\frac{1}{12} M_{E}\left(h_{E}^{2}+l_{E}^{2}\right)\right. \\
& \left.+M_{E}\left(\frac{1}{2} h_{L}+\frac{1}{2} h_{E}\right)^{2}\right) \\
& +2\left(\frac{1}{12} M_{E}\left(h_{E}^{2}+w_{E}^{2}\right)\right. \\
& +M_{E}\left(\left(\frac{1}{2} h_{L}+\frac{1}{2} h_{E}\right)^{2}\right. \\
& \left.\left.+\left(\frac{1}{2} l_{L}+\frac{1}{2} s_{C B}\right)^{2}\right)\right) \\
& +2\left(\frac{1}{12} M_{S M}\left(h_{S M}^{2}+l_{S M}^{2}\right)\right. \\
& \left.+M_{S M}\left(\frac{1}{2} h_{L}+\frac{1}{2} h_{S M}\right)^{2}\right) \\
& +2\left(\frac{1}{12} M_{S M}\left(h_{S M}^{2}+w_{S M}^{2}\right)\right. \\
& +M_{S M}\left(\left(\frac{1}{2} h_{L}+\frac{1}{2} h_{S M}\right)^{2}\right. \\
& \left.\left.+\left(\frac{1}{2} s_{C B}+l_{L}-\frac{1}{2} w_{S M}\right)^{2}\right)\right) \\
& +2\left(\frac{1}{12} M_{L}\left(h_{L}^{2}+l_{L}^{2}\right)\right) \\
& +2\left(\frac{1}{12} M_{L}\left(h_{L}^{2}+w_{L}^{2}\right)\right. \\
& \left.+M_{L}\left(\left(\frac{1}{2} l_{L}+\frac{1}{2} s_{C B}\right)^{2}\right)\right) \\
& +\left(\frac{1}{12} M_{C B}\left(h_{C B}^{2}+s_{C B}^{2}\right)\right) \\
& +\left(\frac{1}{12} M_{B}\left(h_{B}^{2}+w_{B}^{2}\right)\right. \\
& \left.+M_{B}\left(\frac{1}{2} h_{C B}+\frac{1}{2} h_{B}\right)^{2}\right)
\end{aligned}
$$

To determine the coordinates of the $\mathrm{CoG}$ of the components brushless motors (BM), brushless motor mounting (BMM), and propeller $(\mathrm{P})$ on the $x, y$, and $z$-axes, then we can use the method Denavit-Hartenberg which is the compact form of the method forward kinematics [16]. The general transformation matrix ${ }_{j}^{j-1} T$ for a single link can be obtained as shown in (37). 


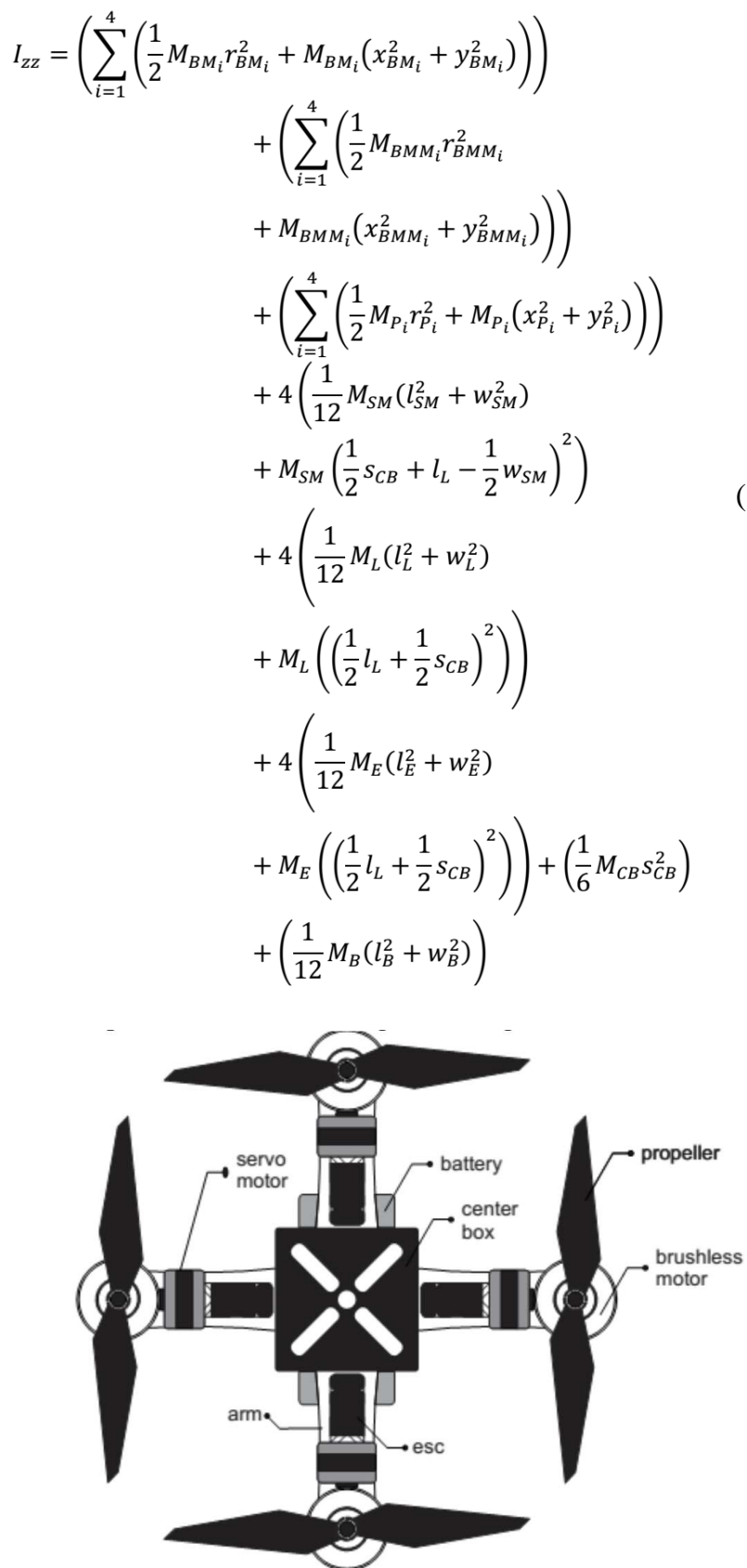

Fig. 5 Quad tiltrotor top view design where $R_{x}$ and $R_{z}$ present rotation, $D_{x}$ and $Q_{j}$ denote translation, the distance from $Z_{j-1}$ to $Z_{j}$ measured along $X_{j-1}$ is assigned as $\mathrm{a}_{j-1}$, the angle between $Z_{j-1}$ and $Z_{j}$ measured about $X_{j}$ is assigned as $\alpha_{j-1}$, the distance from $X_{j-1}$ to $X_{j}$ measured along $Z_{j}$ is assigned as $d_{j}$, the angle between $X_{j-1}$ to $X_{j}$ measured about $Z_{j}$ is assigned as $\theta_{j}, j$ is an endeffector, and $(j-1)$ is a base [17]. An alternative representation of end-effector $T$ can be written as

$$
\text { end-effector } T=\left[\begin{array}{cccc}
r_{11} & r_{12} & r_{13} & p_{x} \\
r_{21} & r_{22} & r_{23} & p_{y} \\
r_{31} & r_{32} & r_{33} & p_{z} \\
0 & 0 & 0 & 1
\end{array}\right]
$$

Then using equation (37) we can obtain transformation matrix for each component, which consists of brushless motors (BM), brushless motor mounting (BMM), and propeller (P) as shown in equation (39), (40), and (41).

$$
\begin{aligned}
& \begin{array}{l}
{ }_{\mathrm{j}}^{\mathrm{j}-1} \mathrm{~T}_{\mathrm{BM}_{\mathrm{i}}}= \\
{\left[\begin{array}{cccc}
\cos 0 & -\sin 0 & 0 & 0 \\
\sin 0 \cos \alpha_{\mathrm{i}} & \cos 0 \cos \alpha & -\sin \alpha_{\mathrm{i}} & \left(-\sin \alpha_{\mathrm{i}}\right)\left(\frac{1}{2} \mathrm{~h}_{\mathrm{BM}}+\mathrm{h}_{\mathrm{BMM}}\right) \\
\sin 0 \sin \alpha_{\mathrm{i}} & \cos 0 \sin \alpha_{\mathrm{i}} & \cos \alpha_{\mathrm{i}} & \left(\cos \alpha_{\mathrm{i}}\right)\left(\frac{1}{2} \mathrm{~h}_{\mathrm{BM}}+\mathrm{h}_{\mathrm{BMM}}\right) \\
0 & 0 & 0 & 1
\end{array}\right]} \\
=\left[\begin{array}{cccc}
1 & 0 & 0 & 0
\end{array}\right] \\
{\left[\begin{array}{cccc}
0 & \cos \alpha & -\sin \alpha_{\mathrm{i}} & \left(-\sin \alpha_{\mathrm{i}}\right)\left(\frac{1}{2} \mathrm{~h}_{\mathrm{BM}}+\mathrm{h}_{\mathrm{BMM}}\right) \\
0 & \sin \alpha_{\mathrm{i}} & \cos \alpha_{\mathrm{i}} & \left(\cos \alpha_{\mathrm{i}}\right)\left(\frac{1}{2} \mathrm{~h}_{\mathrm{BM}}+\mathrm{h}_{\mathrm{BMM}}\right) \\
0 & 0 & 0 & 1
\end{array}\right]}
\end{array} \\
& \begin{array}{l}
{ }_{j}^{j-1} \mathrm{~T}_{\mathrm{BBM}_{\mathrm{i}}}= \\
{\left[\begin{array}{cccc}
\cos 0 & -\sin 0 & 0 & 0 \\
\sin 0 \cos \alpha_{\mathrm{i}} & \cos \theta_{\mathrm{i}} \cos \alpha & -\sin \alpha_{\mathrm{i}} & \left(-\sin \alpha_{\mathrm{i}}\right)\left(\frac{1}{2} \mathrm{~h}_{\mathrm{BMM}}\right) \\
\sin 0 \sin \alpha_{\mathrm{i}} & \cos 0 \sin \alpha_{\mathrm{i}} & \cos \alpha_{\mathrm{i}} & \left(\cos \alpha_{\mathrm{i}}\right)\left(\frac{1}{2} \mathrm{~h}_{\mathrm{BMM}}\right) \\
0 & 0 & 0 & 1
\end{array}\right]}
\end{array} \\
& =\left[\begin{array}{cccc}
1 & 0 & 0 & 0 \\
0 & \cos \alpha & -\sin \alpha_{i} & \left(-\sin \alpha_{i}\right)\left(\frac{1}{2} h_{B M M}\right) \\
0 & \sin \alpha_{i} & \cos \alpha_{i} & \left(\cos \alpha_{i}\right)\left(\frac{1}{2} h_{B M M}\right) \\
0 & 0 & 0 & 1
\end{array}\right] \\
& {\left[\begin{array}{cccc}
\mathrm{j}_{\mathrm{P}_{\mathrm{i}}}= & -\sin 0 & 0 & 0 \\
\sin 0 \cos \alpha_{\mathrm{i}} & \cos \theta_{\mathrm{i}} \cos \alpha & -\sin \alpha_{\mathrm{i}} & \left(-\sin \alpha_{\mathrm{i}}\right)\left(\frac{1}{2} \mathrm{~h}_{\mathrm{P}}+\mathrm{h}_{\mathrm{BM}}+\mathrm{h}_{\mathrm{BMM}}\right) \\
\sin 0 \sin \alpha_{\mathrm{i}} & \cos 0 \sin \alpha_{\mathrm{i}} & \cos \alpha_{\mathrm{i}} & \left(\cos \alpha_{\mathrm{i}}\right)\left(\frac{1}{2} \mathrm{~h}_{\mathrm{P}}+\mathrm{h}_{\mathrm{BM}}+\mathrm{h}_{\mathrm{BMM}}\right) \\
0 & 0 & 0 & 1
\end{array}\right]} \\
& =\left[\begin{array}{ccccc}
0 & 0 & 0 & 0 & 0 \\
0 & \cos \alpha & -\sin \alpha_{\mathrm{i}} & \left(-\sin \alpha_{\mathrm{i}}\right)\left(\frac{1}{2} \mathrm{~h}_{\mathrm{P}}+\mathrm{h}_{\mathrm{BM}}+\mathrm{h}_{\text {BMM }}\right) \\
0 & \sin \alpha_{\mathrm{i}} & \cos \alpha_{\mathrm{i}} & \left(\cos \alpha_{\mathrm{i}}\right)\left(\frac{1}{2} \mathrm{~h}_{\mathrm{P}}+\mathrm{h}_{\mathrm{BM}}+\mathrm{h}_{\text {BMM }}\right) \\
0 & 0 & 0 & 1
\end{array}\right]
\end{aligned}
$$

By using equation (38), (39), (40), and (41), we can obtain the $\mathrm{CoG}$ coordinates of the position of each component of brushless motors (BM), brushless motor mounting (BMM), and a propeller $(\mathrm{P})$ on the $x, y$, and $z$ axes.

\section{RESULTS AND DISCUSSION}

We can use various methods to control a quad tilt-rotor. However, we can improve the control by implementing modern control system. Modern control systems use the concept of state space as shown in equation (42).

$$
\begin{aligned}
& \dot{x}=A x+B u \\
& y=C x+D u
\end{aligned}
$$

where

$$
\begin{aligned}
& \boldsymbol{x}=\text { state } \\
& \boldsymbol{u}=\text { process input } \\
& \boldsymbol{y}=\text { process output } \\
& \boldsymbol{A}=\text { state derivative and state relation matrix }
\end{aligned}
$$


$\boldsymbol{B}=$ state derivative and process input relation matrix

$\boldsymbol{C}=$ output process and state relation matrix

$\boldsymbol{D}=$ output and input process relation matrix

By using the modelling of the equations (17), (18), and (12) representing the movement of translation in the $x_{n} y$, and $z$ respectively, we can tune create a state space model system of quad tiltrotor. Similarly, for rotation motion, we can take advantage of the equation (21), (22), and (33). By utilizing a quad tilt-rotor prototype shown in Fig. 6, we tried to simulate by filling entire components of these equations using the original data from the prototype.

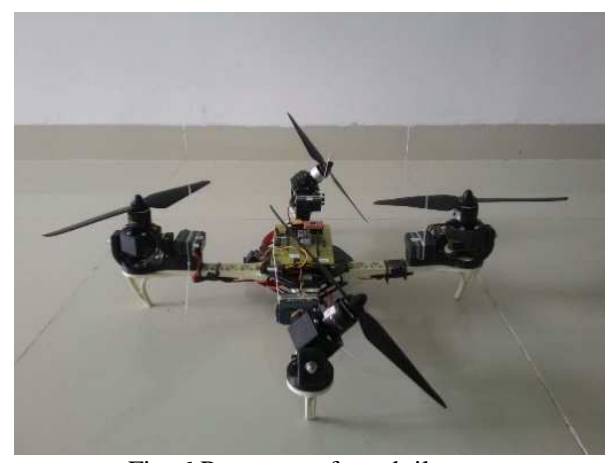

Fig. 6 Prototype of quad tiltrotor

Before doing the simulation, we must determine the constant thrust $\mathrm{b}$ brushless motors and brushless motors the torque constant $k$ values using equation (8) and (32) respectively. The process of calculating the constants starting with determining the constants $C_{F}$ and $C_{T}$ [14]. In this research, we use $b=1.32 \times 10^{-4}$ and $k=6.83 \times 10^{-6}$.

We can apply control method on systems that have more than one input and output of each (MIMO or Multi Input Multi Output). It will be advantages if we compare to classical control methods such as PID control method that can only process a single input and a single output (SISO). PID control system can still handle the MIMO system, but we do it sequentially [18]. By changing the equation model (34), (35), and (29) representing the movement of translation in the $x, y$, and $z$ axes respectively and the equation (36), (37), and (30) representing the movement of rotation forming a roll $\phi$, pitch $\theta$, and yaw $\psi$, we can make the state space equation as shown as follows:on systems that have the input and output of each of more than one (MIMO or Multi Input Multi Output). The state space equation of quad tilrotor system consists of $\boldsymbol{x}, \boldsymbol{u}, \boldsymbol{y}, \boldsymbol{A}, \boldsymbol{B}, \boldsymbol{C}$, and $\boldsymbol{D}$ matrix as shown in equation (43) - (47).

$$
\boldsymbol{x}=\left[\begin{array}{c}
x \\
v_{x} \\
y \\
v_{y} \\
z \\
v_{z} \\
\phi \\
\omega_{\phi} \\
\theta \\
\omega_{\theta} \\
\psi \\
\omega_{\psi}
\end{array}\right], \dot{\boldsymbol{x}}=\left[\begin{array}{c}
\dot{x} \\
v_{x} \\
\dot{y} \\
v_{y} \\
\dot{z} \\
\dot{v}_{z} \\
\dot{\phi} \\
\dot{\omega}_{\phi} \\
\dot{\theta} \\
\dot{\omega}_{\theta} \\
\dot{\psi} \\
\dot{\omega_{\psi}}
\end{array}\right], \boldsymbol{u}=\left[\begin{array}{l}
u_{1} \\
u_{2} \\
u_{3} \\
u_{4} \\
u_{5} \\
u_{6}
\end{array}\right]=\left[\begin{array}{l}
F_{1} \\
F_{2} \\
F_{3} \\
\tau_{1} \\
\tau_{2} \\
\tau_{3}
\end{array}\right], \boldsymbol{y}=\left[\begin{array}{l}
y_{1} \\
y_{2} \\
y_{3} \\
y_{4} \\
y_{5} \\
y_{6}
\end{array}\right]=\left[\begin{array}{l}
x \\
y \\
z \\
\phi \\
\theta \\
\psi
\end{array}\right]
$$

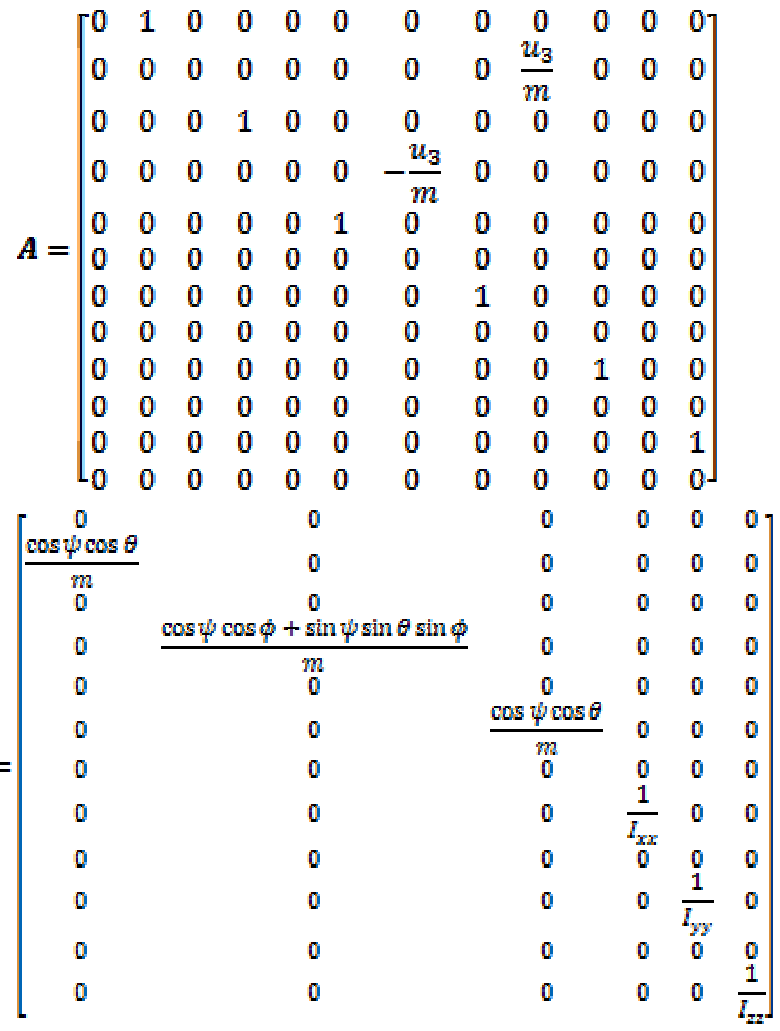

$$
\begin{aligned}
& C=\left[\begin{array}{llllllllllll}
1 & 0 & 0 & 0 & 0 & 0 & 0 & 0 & 0 & 0 & 0 & 0 \\
0 & 0 & 1 & 0 & 0 & 0 & 0 & 0 & 0 & 0 & 0 & 0 \\
0 & 0 & 0 & 0 & 1 & 0 & 0 & 0 & 0 & 0 & 0 & 0 \\
0 & 0 & 0 & 0 & 0 & 0 & 1 & 0 & 0 & 0 & 0 & 0 \\
0 & 0 & 0 & 0 & 0 & 0 & 0 & 0 & 1 & 0 & 0 & 0 \\
0 & 0 & 0 & 0 & 0 & 0 & 0 & 0 & 0 & 0 & 1 & 0
\end{array}\right] \\
& D=\left[\begin{array}{llllll}
0 & 0 & 0 & 0 & 0 & 0 \\
0 & 0 & 0 & 0 & 0 & 0 \\
0 & 0 & 0 & 0 & 0 & 0 \\
0 & 0 & 0 & 0 & 0 & 0 \\
0 & 0 & 0 & 0 & 0 & 0 \\
0 & 0 & 0 & 0 & 0 & 0
\end{array}\right]
\end{aligned}
$$

where

$$
\begin{aligned}
& u_{1}=b\left(\omega_{2}^{2} \sin \alpha_{2}+\omega_{4}^{2} \sin \alpha_{4}\right) \\
& u_{2}=b\left(\omega_{1}^{2} \sin \alpha_{1}+\omega_{3}^{2} \sin \alpha_{3}\right) \\
& u_{3}=b\left(\omega_{1}^{2} \cos \alpha_{1}+\omega_{3}^{2} \cos \alpha_{3}+\omega_{2}^{2} \cos \alpha_{2}+\omega_{4}^{2} \cos \alpha_{4}\right) \\
& u_{4}=b l\left(\omega_{2}^{2} \cos \alpha_{2}-\omega_{4}^{2} \cos \alpha_{4}\right)+k\left(\omega_{2}^{2} \sin \alpha_{2}+\omega_{4}^{2} \sin \alpha_{4}\right) \\
& u_{5}=b l\left(\omega_{1}^{2} \cos \alpha_{1}-\omega_{3}^{2} \cos \alpha_{3}\right)+k\left(\omega_{1}^{2} \sin \alpha_{1}+\omega_{3}^{2} \sin \alpha_{3}\right) \\
& u_{6}=k\left(\omega_{1}^{2} \cos \alpha_{1}-\omega_{2}^{2} \cos \alpha_{2}+\omega_{3}^{2} \cos \alpha_{3}-\right. \\
& \left.\omega_{4}^{2} \cos \alpha_{4}\right)+b l\left(\omega_{4}^{2} \sin \alpha_{4}-\omega_{2}^{2} \sin \alpha_{2}+\omega_{3}^{2} \sin \alpha_{3}-\right. \\
& \left.\omega_{1}^{2} \sin \alpha_{1}\right)
\end{aligned}
$$

By using the concept of full state feedback [19] [20], we can design a close loop control in state space system. Then we apply this concept by using $\boldsymbol{K}$ as feedback gain [21].

Before we calculate the $\boldsymbol{K}$ gain, we design the control simulation using the concept of full state feedback in state space system as shown in Fig. 7. $\boldsymbol{K}$ gain can act as the performance regulator matrix of the system. Input process feedback is influenced by $\boldsymbol{K}$ gain as shown in equation (48).

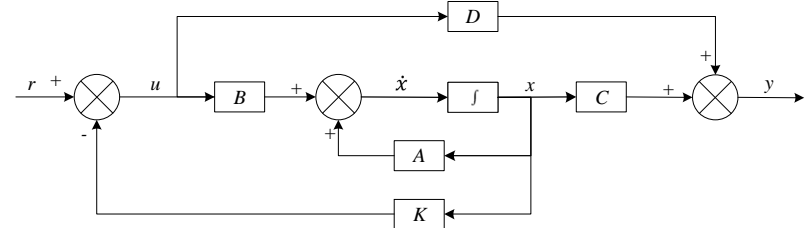

Fig. 7 Control system design with K gain [22] 


$$
\boldsymbol{u}=\boldsymbol{K} \boldsymbol{x}
$$

We can create the simulation diagram block in tools like Simulink based on diagram block in Fig. 7 and state space system that we have made before.

Fig. 8 shows the model simulation of quad tiltrotor.

The simulation model is divided into four pieces parts, consisting of:

- State space with full state feedback

- Flight Scenario

- Process Noises

- Force and Torque Conversion to Brushless DC (BLDC) Motors and Servos Rotation

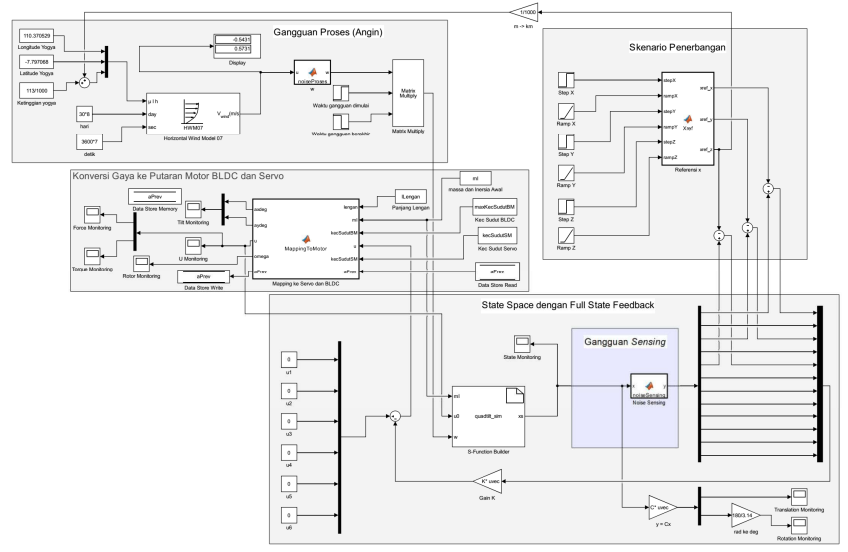

Fig. 8 Quad tiltrotor model simulation using Simulink

The state space with full state feedback is the central part of the system. This section is designed using the model of equation (6.2). Then the flight scenario mission arrangement consists of setting the quad tiltrotor movement direction. Flight is regulated by 3-dimensional motion directions, specifically the $x, y$ and $z$-axes.

Process noises contribute to give some white noise (interference) on the system. These disturbances are generated randomly but still in the range that we have determined at specified intervals. Force conversion to a brushless motor (BLDC) and servo rotations represents the performance of a vehicle based on the model that we have simulated.

We can set the $\boldsymbol{K}$ gain matrix value using various methods. Some example of the methods are manual tuning, ANN [23], Pole Placement [24], Ackermann [25], LQR [26], etc.

The LQR (Linear Quadratic Regulator) controller is a development of the Hamilton-Jacobi method that utilizes the $\mathrm{K}$ matrix as a gain in full state feedback as shown in Fig. 7. Systems that do not have a control signal reference are referred to as regulatory systems.

The gain matrix K can be obtained using equation (49).

$$
K=R^{-1} B^{T} P
$$

where the matrix $\mathrm{P}$ is derived from Riccati equation according to equation (50).

$$
A^{T} P+P A-P B R^{-1} B^{T} P+Q=0
$$

The matrix selection of $\boldsymbol{Q}$ and $\boldsymbol{R}$ elements is guided by:

1. The greater of $\boldsymbol{Q}$, the greater the cost of strengthening $\boldsymbol{K}$, thus speeding up the system to achieve an intermediate state.

2. The larger of $\boldsymbol{R}$, it will reduce the cost of strengthening $\boldsymbol{K}$ and make settling state process slower.

After getting the $\boldsymbol{K}$ gain matrix value as shown in equation (51), we can run the simulation. The simulation results are shown in

Fig. 9 - Fig. 14.
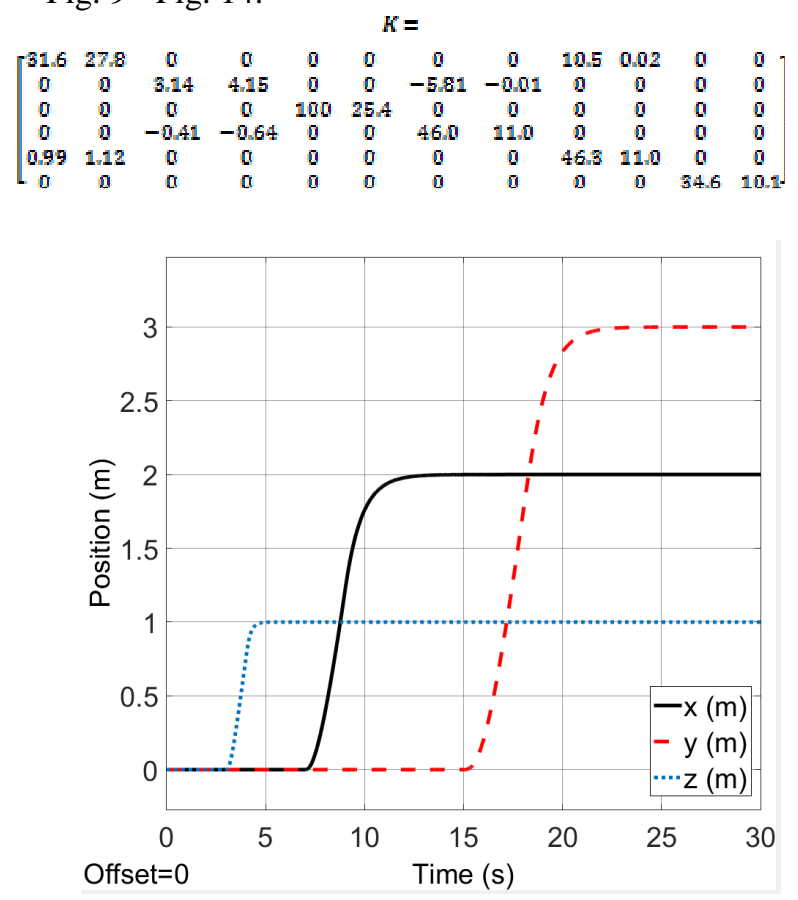

Fig. 9 Translation Movement Simulation Results

Fig. 9 shows the quad tiltrotor translation movement along $x, y$, and $z$-axes based on the scenario that we have created before. There are no overshoots on each of the translation movement curves in the figure. In rotation motion simulation results that shown in Fig. 10, we can see that there are slight overshoots in each rotation. The overshoots are in the range $10^{3}$. We can say that the feedback gain $\boldsymbol{K}$ has worked well in overshoot damping.

Based on the scenario that we created, the system set how strong the force in each axis are automatically. Due to the force and torque are the control inputs $\boldsymbol{u}$, then they are influenced by the gain $\boldsymbol{K}$ as shown in equation (48). It is intended that the system can minimize the overshoots and keep response times as desired. We can see the results of force and torque on each axis in Fig. 11 and Fig. 12 respectively. 


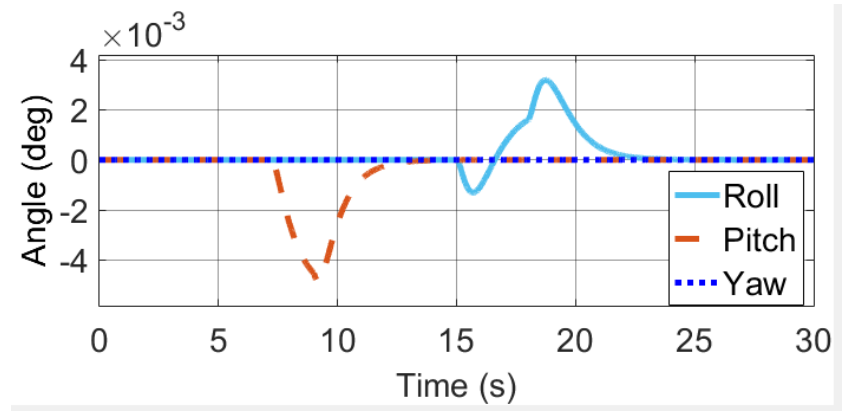

Fig. 10 Rotation Motion Simulation Results
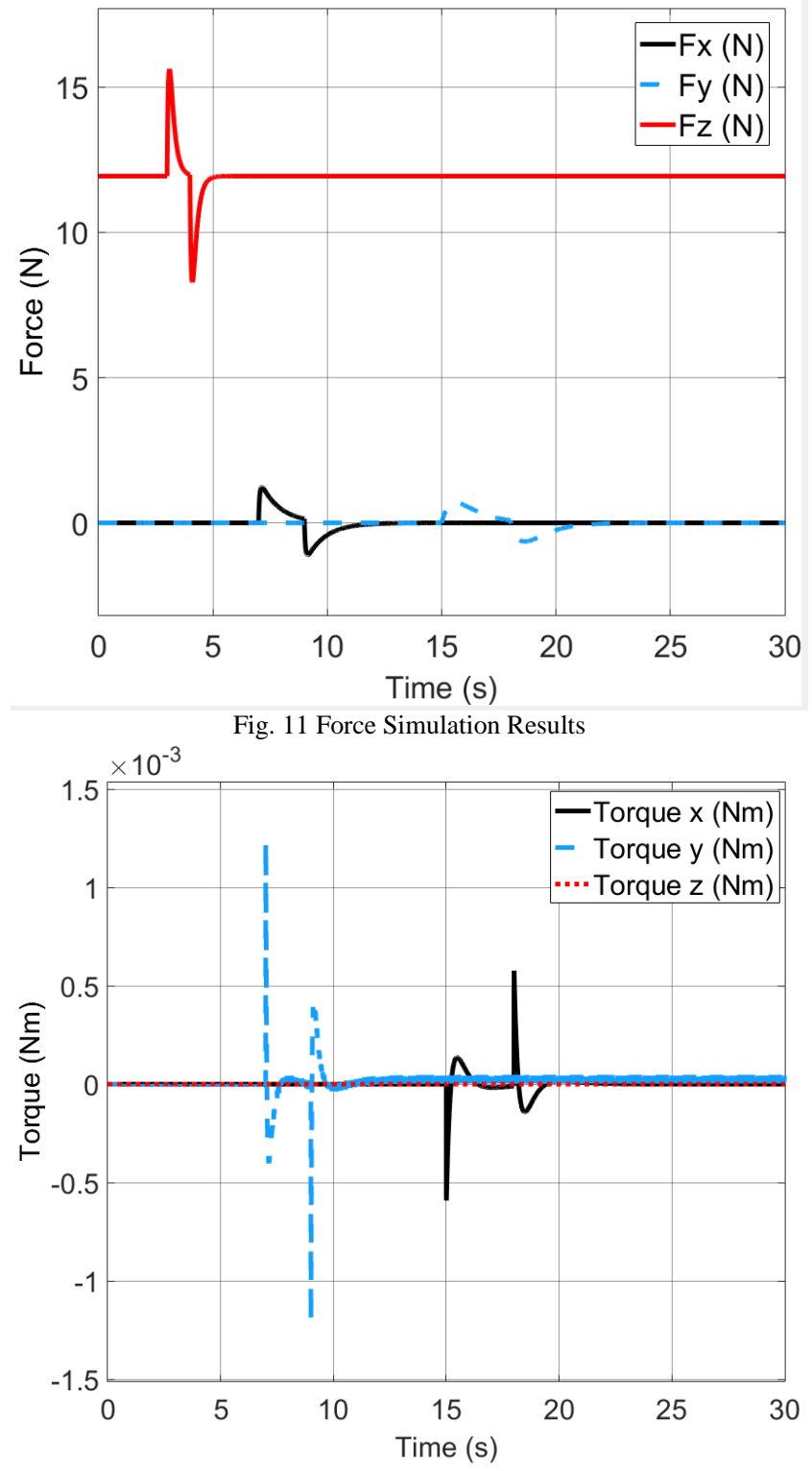

Fig. 12 Torque Simulation Results

Fig. 11 shows that the initial value of the force on the $\mathrm{z}$ axis (vertical) is in the range 12 Newton. This value is equal to the weight of the quad tiltrotor. It explains that the system is an equilibrium state. Then at the time shows 3 seconds, the power of vertical force $\left(F_{z}\right)$ begins to rise to about 16 Newton. This condition indicates that a quad tiltrotor takes off to a height of 1-meter accordance with the flight scenario. Upon approaching the desired height, the force of the vertical drop to about 8 Newton. It is intended that no overshoot is exceeding the height we want. Having almost reached the desired height, the power of the vertical force rises again until it reaches the equilibrium value (12 Newton). The power of force on the $x$-axis $\left(F_{x}\right)$ and $y$-axis $\left(F_{y}\right)$ rise and fall accordance with the scenario quad tiltrotor flight wanting to move along the $x$ and $y$-axes.

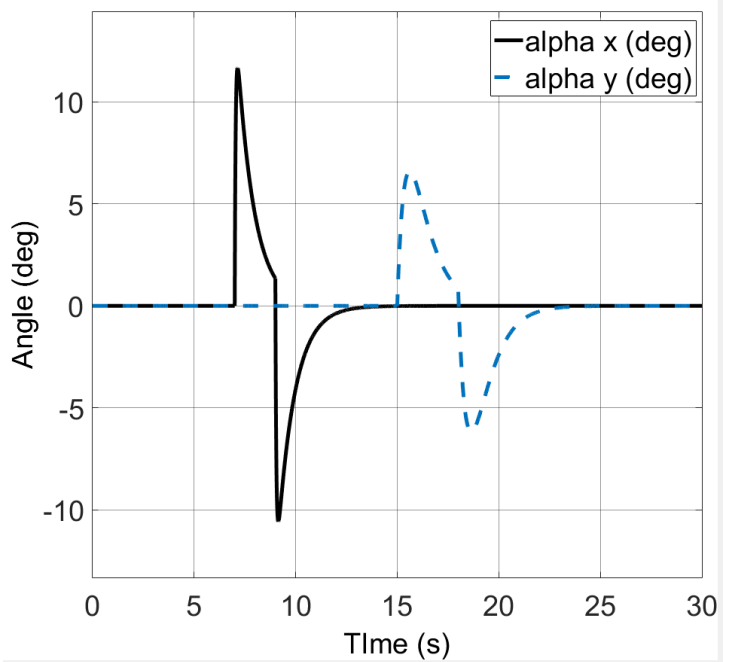

Fig. 13 Tilting Rotor Simulation Results

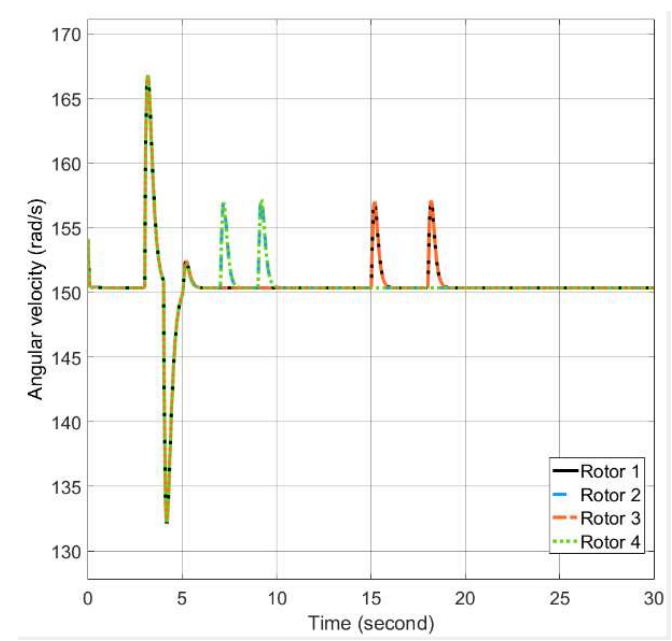

Fig. 14 Rotors Angular Velocity Simulation Results

In Fig. 12, we can see the strength of torque around the $x$ and $y$-axes do not change significantly (only around $10^{-3}$ ). This condition is due to the torque appears as an attempt to dampen overshoot in the rotational motion.

Fig. 13 shows the change of each rotor tilting angle in the $x$ and $y$-axes. The system arranges the changes of this tilting according to the equation (23). The system then adjusts the rotational velocity of each rotor appearing unwanted force. This undesirable force can lead to misguided quad tiltrotor flight. Fig. 14 shows the results of each rotors rotating speed setting.

\section{CONCLUSION}

The quad tilt rotor mathematical models help us in designing a control system which is good for the system. We can apply a variety of methods to control quad tiltrotor running well. The simulations show that the quad tiltrotor 
successful translational motion without significant interference. Also, undesirable rotation movement in the quad tiltrotor flight when performing the translational motions resulting from the transition process associated with the tilt rotor change was successfully reduced below 1 degree. However, we need a control system that is robust, even robust adaptive, if we want to create an autonomous flight. As one method of robust control, the modern control can be expanded by adding a tracking system. However, the tracking system can usually lead to their steady-state error. To overcome this, we need the concept of integrator as the eliminator of the steady-state error. To get to the robust adaptive systems, we can use Kalman Filter algorithm as an estimator and Lyapunov stability method.

\section{REFERENCES}

[1] E. A. Euteneuer and G. Papageorgiou, "UAS insertion into commercial airspace: Europe and US standards perspective," in IEEE/AIAA 30th Digital Avionics Systems Conference, 2011, p. 5C5-1-5C5-12.

[2] S. Gupte, Paul Infant Teenu Mohandas, and J. M. Conrad, "A survey of quadrotor Unmanned Aerial Vehicles," in 2012 Proceedings of IEEE Southeastcon, 2012, pp. 1-6.

[3] S. Bouabdallah, M. Becker, and R. Siegwart, "Autonomous Miniature Flying Robots: Coming Soon! - Research, Development, and Results," IEEE Robot. Autom. Mag., vol. 14, no. 3, pp. 88-98, Sep. 2007.

[4] K. T. Oner, E. Cetinsoy, M. Unel, M. F. Aksit, I. Kandemir, and K. Gulez, "Dynamic Model and Control of a New Quadrotor Unmanned Aerial Vehicle with Tilt-Wing Mechanism," Int. J. Mech. Aerospace, Ind. Mechatronics Eng., vol. 2, no. 9, pp. 12-17, 2008.

[5] R. Voyles and G. Jiang, "Hexrotor UAV Platform Enabling Dextrous Interaction with Structures - Preliminary Work," in IEEE International Symposium on Safety, Security, and Rescue Robotics (SSRR), 2012, vol. 0, no. c, pp. 1-7.

[6] A. Sanchez, J. Escareño, O. Garcia, and R. Lozano, "Autonomous Hovering of a Noncyclic Tiltrotor UAV : Modeling, Control and Implementation," in Proceedings of the 17th World Congress The International Federation of Automatic Control, 2008, pp. 803-808.

[7] F. Forte, R. Naldi, A. Serrani, and L. Marconi, "Control of Modular Aerial Robots: Combining Under and Fully Actuated Behaviors," in IEEE 51st IEEE Conference on Decision and Control (CDC), 2012, pp. $1160-1165$.

[8] M. D. Hua, T. Hamel, and C. Samson, "Control of VTOL Vehicles with Thrust-direction Tilting," in The 19th World Congress of the International Federation of Automatic Control, 2014.

[9] A. Dharmawan, A. Ashari, and A. E. Putra, "Quadrotor flight stability system with Routh stability and Lyapunov analysis," in AIP Conf. Proc. 1755, 170007 (2016), 2016, vol. 1755, p. 170007.
[10] M. Santos, V. Lopez, and F. Morata, "Intelligent Fuzzy Controller of a Quadrotor," in IEEE International Conference on Intelligent Systems and Knowledge Engineering, 2010, pp. 141-146.

[11] T. K. Priyambodo, A. E. Putra, and A. Dharmawan, "Optimizing control based on ant colony logic for Quadrotor stabilization," in 2015 IEEE International Conference on Aerospace Electronics and Remote Sensing Technology (ICARES), 2015, vol. 1, pp. 1-4.

[12] A. Z. Azfar and D. Hazry, "A Simple Approach on Implementing IMU Sensor Fusion in PID Controller for Stabilizing Quadrotor Flight Control," in 2011 IEEE 7th International Colloquium on Signal Processing and its Applications, 2011, pp. 28-32.

[13] M. Ryll, H. H. Bülthoff, and P. R. Giordano, "A novel overactuated quadrotor unmanned aerial vehicle: Modeling, control, and experimental validation," IEEE Trans. Control Syst. Technol., vol. 23, no. 2, pp. 540-556, Mar. 2015.

[14] R. W. Prouty, Helicopter Performance, Stability, and Control, 2002nd ed. Krieger Pub Co, 2002.

[15] R. C. Hibbeler, Dynamics. Hoboken, New Jersey: Pearson Prentice Hall, 2016.

[16] S. Kucuk and Z. Bingul, Robot Kinematics - Forward and Inverse Kinematics. Germany: Pro Literatur Verlag, Germany / ARS, Austria, 2007.

[17] J. J. Craig, Introduction to Robotics Mechanics and Control, Third. New Jersey, USA: Pearson Prentice Hall, 2005.

[18] K. Ogata, Modern Control Engineering, 5th ed. New Jersey, USA: Prentice-Hall, 2010.

[19] M. Athans and P. Falb, Optimal Control: An Introduction to the Theory and Its Applications (Dover Books on Engineering). New York, USA: Dover Publications, 2006.

[20] R. E. Bellman, Dynamic Programming. New Jersey, USA: Dover Publications, 2003.

[21] E. Lavretsky and K. A. Wise, Robust and Adaptive Control. London: Springer London, 2013.

[22] B. Messner and D. Tilbury, "Control Tutorials for Matlab and Simulink," 2011. [Online]. Available: http://ctms.engin.umich.edu/CTMS/index.php?aux=Home.

[23] J. F. Shepherd and K. Tumer, "Robust neuro-control for a micro quadrotor," in Proceedings of the 12th annual conference on Genetic and evolutionary computation - GECCO '10, 2010, p. 1131.

[24] E. Pfeifer and F. Kassab Jr., "Dynamic Feedback Controller of an Unmanned Aerial Vehicle," in 2012 Brazilian Robotics Symposium and Latin American Robotics Symposium, 2012, pp. 261-266.

[25] C. Di, Q. Geng, Q. Hu, and W. Wu, "High performance L1 adaptive control design for longitudinal dynamics of fixed-wing UAV," in The 27th Chinese Control and Decision Conference (2015 CCDC), 2015, vol. 1, no. 1, pp. 1514-1519.

[26] A. Dharmawan, A. Ashari, and A. E. Putra, "PID Control Systems Using LQR Approach for Quadrotor Flight Stability," in International Conference on Science and Technology (ICST), 2016. 